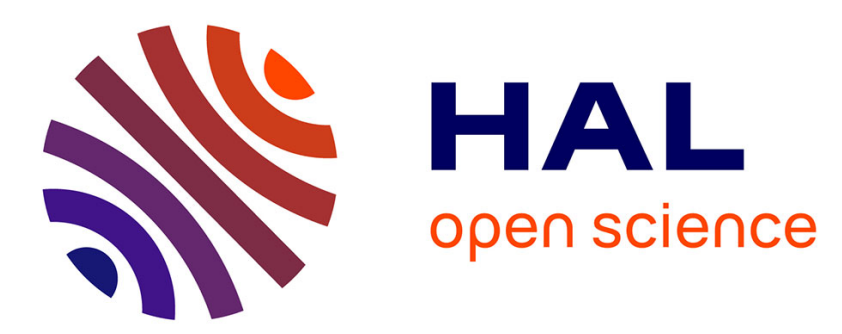

\title{
A Noise Spectroscopy-Based Selective Gas Sensing with MOX Gas Sensors
}

S. Gomri, J. Seguin, T. Contaret, T. Fiorido, Khalifa Aguir

\section{To cite this version:}

S. Gomri, J. Seguin, T. Contaret, T. Fiorido, Khalifa Aguir. A Noise Spectroscopy-Based Selective Gas Sensing with MOX Gas Sensors. Fluctuation and Noise Letters, 2018, 17 (02), pp.1850016. 10.1142/S0219477518500165 . hal-02114914

\section{HAL Id: hal-02114914 \\ https://hal-amu.archives-ouvertes.fr/hal-02114914}

Submitted on 30 Apr 2019

HAL is a multi-disciplinary open access archive for the deposit and dissemination of scientific research documents, whether they are published or not. The documents may come from teaching and research institutions in France or abroad, or from public or private research centers.
L'archive ouverte pluridisciplinaire HAL, est destinée au dépôt et à la diffusion de documents scientifiques de niveau recherche, publiés ou non, émanant des établissements d'enseignement et de recherche français ou étrangers, des laboratoires publics ou privés. 


\title{
A Noise Spectroscopy-Based Selective Gas Sensing with MOX Gas Sensors
}

\author{
S. Gomri**, J. Seguin ${ }^{\dagger}$, T. Contaret ${ }^{\dagger}$, T. Fiorido ${ }^{\dagger}$ and K. Aguir ${ }^{\dagger}$ \\ ${ }^{*}$ National School of Engineers of Sfax \\ Road of Soukra, Km 4. 3000, Tunisia \\ ${ }^{\dagger}$ Aix Marseille University, University Toulon \\ CNRS, IM2NP, Marseille, France \\ \$sami.gomri14@gmail.com
}

\begin{abstract}
We propose a new method for obtaining a fluctuation-enhanced sensing (FES) signature of a gas using a single metal oxide (MOX) gas micro sensor. Starting from our model of adsorptiondesorption (A-D) noise previously developed, we show theoretically that the product of frequency by the power spectrum density (PSD) of the gas sensing layer resistance fluctuations often has a maximum which is characteristic of the gas. This property was experimentally confirmed in the case of the detection of $\mathrm{NO}_{2}$ and $\mathrm{O}_{3}$ using a $\mathrm{WO}_{3}$ sensing layer. This method could be useful for classifying gases. Furthermore, our noise measurements confirm our previous model showing that PSD of the A-Dnoise in MOX gas sensor is a combination of Lorentzians having a low frequency magnitude and a cut-off frequency which depends on the nature of the detected gas.
\end{abstract}

Keywords: Metal oxide gas micro sensor; adsorption-desorption noise; fluctuation-enhanced sensing; noise spectroscopy; gas sensor selectivity.

\section{Introduction}

Many applications such as monitoring the environment or controlling of some industrial process, require selective and sensitive gas sensors. Metal oxide (MOX) gas sensors are one of the most popular types because of high sensitivity, stability, and compatibility with planar technology [1]. However, it is well known that MOX gas sensors are not selective to detect a single chemical species in a gaseous mixture [2]. This lack of selectivity is an obstacle to the development of high-quality semiconducting metal-oxide-based chemical detectors.

Intensive search of new materials and new techniques for increasing MOX-based gas sensors selectivity is of great interest. Fluctuation-enhanced sensing (FES) is one 
solution that can improve the selectivity of gas sensors [3-12]. This experimental technique is based on analysis of the power spectrum density (PSD) of noise fluctuations measured at the terminals of sensors in the presence of one or more gas. The principle of this technique is that the interaction between a gas sensor and the detected gas molecule is always a dynamic process [5-7]. Fluctuations that result from this interaction carry a "stochastic fingerprint" of the chemicals interacting with the sensor. The stages following the sensor in a FES system typically contain filters and preamplifier(s) to extract and amplify the stochastic signal components. Then, the selected statistical properties of the amplified noise are analyzed, and a corresponding pattern is generated as the stochastic fingerprint of the sensed agent.

FES technique might be highly useful for improving gas sensors' selectivity if both theoretical and experimental studies can be adequately developed with the aim of seeking a correlation between the noise spectrum and the nature of the detected gas. Behind this study, finding parameters for gases' identification is a major issue. They form the signature of the detected gas, also known as "FES signatures".

In a recent work [18], we developed a theoretical modeling of noise in polycrystalline MOX gas sensor. We have shown that the measured noise spectra is a combination of multi-Lorentzian components and that the origin of the noise is the adsorption-desorption (A-D) process on the surface of grains which constitutes the sensitive layer. The dependence of Lorentzian parameters with gas type has been clearly demonstrated. From this model, we present the theoretical study of a new method for the identification of gases and its application to the discrimination between $\mathrm{O}_{3}$ and $\mathrm{NO}_{2}$ using a single micro sensor.

\section{Noise in Gas Sensors}

\subsection{Applications of noise spectroscopy to gas sensors}

Usually, noise in sensor devices is taken as unfavorable factor. However, many theoretical and experimental works [3-19] have shown that the electrical noise generated in gas sensor can be a useful signal for improving the sensor's selectivity. This goal can be reached by measuring and analyzing the fluctuations of the sensor signal called sensor noise. Superficial examination identifies chemical sensor fluctuations as conventional noise, while, in fact, this stochastic component contains an important part of the chemical signal. This part of the sensor noise is used and identified as a signal which is related to the exposure to the sensed chemical. There are two main noise sources that are linked to the chemical environment.

The first source is due to the diffusion of the sensed gas molecules on the sensor surface. That is, at the molecular level, the diffusive motion of the gas molecule is a random walk. These diffusive motions can lead to various diffusive fluctuation phenomena which can dominate the fluctuation at low frequencies [10, 13].

The second source is related to the occupation of the sensing layer by the sensed gas molecules. That is, at the microscopic level, molecular adsorption and desorption is a stochastic process due to the random aspect of the residence and the absence 
times. This noise is called A-D noise [14-21]. In these works, noise spectroscopy has been proposed as a mean of extracting a more selective response from chemiresistive and surface acoustic wave gas sensors.

\subsection{Noise modeling}

In MOX gas sensors, the sensing material is a semi-conducting oxide $\left(\mathrm{SnO}_{2}\right.$, $\left.\mathrm{WO}_{3} \ldots\right)$. Thus, the physical origin of noise sources is related to the properties of the metal-oxide sensing layer and the chemical environment. The noise behavior of these devices is expected to result from the superposition of several fluctuation sources.

In MOX, the electronic charge transport is mainly related to the presence or absence of oxygen at a lattice site [22]. Thus, weak fluctuations of local oxygen density can cause appreciable conductance fluctuations. The noise depends strongly on the oxygen stoichiometry and oxygen motion. The A-D process of oxygen atoms, the presence of defects and grain boundaries in metal-oxide cause fluctuations of the oxygen density and, thus fluctuations of the sensing layer electrical conductance. In a gaseous environment, the sensing layer conductance fluctuations due to free carrier's number and mobility fluctuation are related to concentration and distribution fluctuations of adsorbed chemical species. Moreover, in several adsorption theories $[23,24]$, it was shown that the adsorbed-gas concentration at the A-D equilibrium depends on the nature of the gas. So, as gas sensing mechanisms in MOX gas sensors involve reactions between charged oxygen species and target gas, one can expect a coupling between the nature of the detected gas and its effect on the intrinsic noise of the sensing layer. That means, it is expected to exist as a correlation between the nature of the detected gas and the noise generated in the sensing layer.

In an earlier work [18], we presented a model of A-D noise in MOX gas sensors, developing the idea that the fluctuation of the gas sensor resistance is, among others noise sources, due to the fluctuation of the density of gas molecules on the surface of the sensing film. The modeling was developed by taking into account the polycrystalline structure of the sensing layer and the effect of the adsorbed molecule's density fluctuation on the grain boundary barrier height.

The starting idea is that, in a polycrystalline sensing layer, the resistance is mainly due to the contributions of neck resistance $R_{N}$ and grain boundary resistance $R_{\mathrm{GB}}$ [25] which, both, depend on the height of the potential barrier $V_{B}$ and the depletion region width $w$ created at grain boundaries by the gas adsorption (see Fig. 1). Assuming, to simplify the model, that there are as many grain boundaries as necks in the sensing layer, the total sensing layer resistance $R_{\text {sensor }}$ is the summation of all elemental resistances $\left(R_{e}=R_{\mathrm{GB}}+R_{N}\right)$ forming the sensing layer.

The calculation principle of the sensing layer resistance fluctuation is based on the fact that, at the A-D equilibrium, the density of adsorbed gas molecules fluctuates around its A-D equilibrium value [14-18]. Therefore, the potential barrier height $V_{B}$ and the depletion region width $w$ fluctuate too, since they depend on the density of adsorbed gas molecules [18]. Then, the neck resistance $R_{N}$ and the grain boundary 


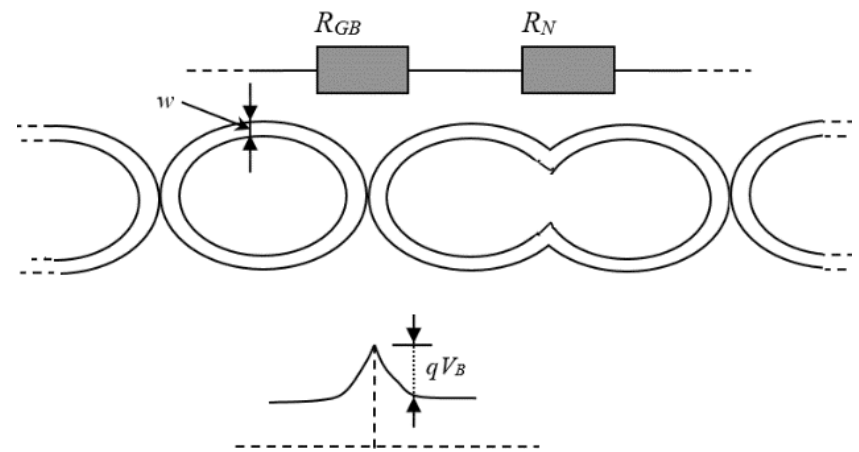

Fig. 1. Neck-grain boundary connection and resistance distribution with the energy-band diagram [25] ( $\boldsymbol{w}$ is the depletion region width induced by the gas adsorption).

resistance $R_{\mathrm{GB}}$ will fluctuate around their A-D equilibrium values since they depend on $V_{B}$ and $w$. Consequently, the elemental resistance $\left(R_{e}=R_{\mathrm{GB}}+R_{N}\right)$ will fluctuate around its A-D equilibrium value. Therefore, the total sensing layer resistance $R_{\text {sensor }}$ fluctuates too.

Using Wolkenstein's isotherm in the case of dissociative and non-dissociative chemisorption [23, 24], by solving the Poisson's equation in spherical coordinates, we derived an exact expression for power spectrum density (PSD) of the A-D noise generated around one grain. With this calculation we have shown that the PSD of the fluctuation of the elemental resistance $\left(R_{e}=R_{\mathrm{GB}}+R_{N}\right)$ is a Lorentzian having a cut-off frequency and a low frequency magnitude depending on the nature of the detected gas, the grains size, and the gas concentration.

Assuming a sensing layer formed by grains with different sizes, we showed that the overall chemisorption-induced noise spectra is the sum of all these Lorentzian components obtained on each grain (see Fig. 3). Bearing in mind that, for the same gas and the same concentration, all grains having the same size give the same spectrum, we have shown that the PDS of the total sensing layer resistance is expressed by [18] as

$$
S_{\delta R_{\text {sensor }}}(f)=\sum_{i=1}^{g} S_{i} \frac{1}{1+\left(\frac{f}{f_{c i}}\right)^{2}},
$$

where $g$ is the number of most prevalent grain sizes involved in the sensing layer. $S_{i}$ and $f_{c i}$ are, respectively, the noise level and the cut-off frequency of the Lorentzian number $i$. Their expressions are given in [18], and they depend on the nature of the detected gas, and on the grain size.

Hence, we have obtained the expression of the fluctuation of the gas sensor resistance. However, in order to check the consistency of the model of the resistance fluctuation, with the measured gas sensor noise, we have to find the relationship between the expression of the total measured noise while the gas sensor is biased, and the expression of the theoretical model given by Eq. (2). 
Indeed, when we measure the noise in the gas sensor, this device must be biased. In this case, the measured noise will take into account the contribution of Johnson noise (thermal noise) generated by the sensing layer resistance. Furthermore, depending on the experimental setup, the measured noise is converted either into a current noise or into a voltage noise. Thus, the PSD of the total noise generated in the gas sensor resistance is expressed by

$$
S_{\text {sensor-noise }}(f)=S_{\text {A-D.sensor-noise }}(f)+S_{\text {th-noise }}(f),
$$

where $S_{\mathrm{A}-\mathrm{D} . \text { sensor-noise }}$ is the PSD of the A-D noise generated in the gas sensor resistance and converted into a current noise or into a voltage noise, and $S_{\text {th-noise }}$ is the PSD of the thermal noise (Johnson noise) generated by the sensing layer resistance. This kind of noise is a white noise, its power density spectrum depends only on the temperature and the value of the sensing layer resistance. It is independent of the nature of the detected gas.

If the gas sensor resistance is biased by a voltage $V_{0}$ and the measured noise is a current fluctuation, the PSD of the fluctuations of the total terminal current across the gas sensor resistance which was expressed by "Eq. (2)" is rewritten by [18]

$$
S_{\delta I_{\text {sensor }}}(f)=\frac{V_{0}^{2}}{R_{\text {sensor }}^{4}} \sum_{i=1}^{g} S_{i} \frac{1}{1+\left(\frac{f}{f_{c i}}\right)^{2}}+\frac{4 k T}{R_{\text {sensor }}},
$$

where $R_{\text {sensor }}$ is the sensing layer resistance, $T$ is the temperature and $k$ is the Boltzman constant.

If the gas sensor resistance is biased by a current $I_{0}$ and the measured noise is a voltage fluctuation, the PSD of the fluctuations of the total voltage across the gas

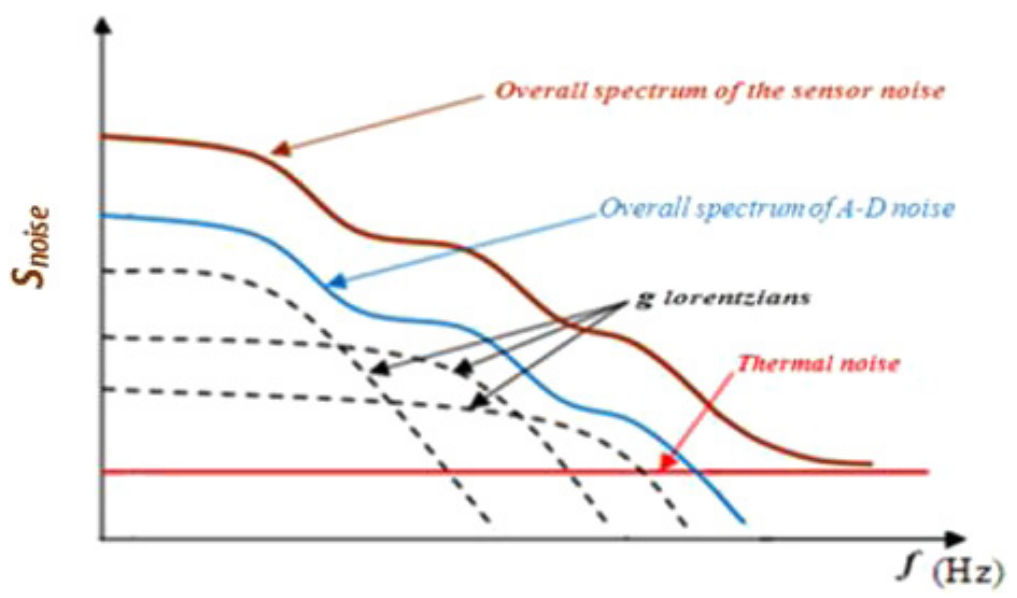

Fig. 2. Decomposition of the overall chemisorption-induced A-D noise spectrum into Lorentzians and the contribution of the thermal noise to the overall spectrum of the sensor noise [18]. 
sensor resistance which was expressed by "Eq. (2)" is rewritten by [18]

$$
S_{\delta V_{\text {sensor }}}(f)=I_{0}^{2} \sum_{i=1}^{g} S_{i} \frac{1}{1+\left(\frac{f}{f_{c i}}\right)^{2}}+4 k T R_{\text {sensor }} .
$$

Hence, with Eqs. (3) and (4), we have shown that at low frequencies the A-D noise will dominate. Whereas, at high frequencies its will be dominated by a white noise due to the thermal noise induced by the sensing layer resistance. It is worth noting that it is only the A-D noise (which is expressed by the first term of the second member of Eqs. (3) and (4)) that depends on the nature of the detected gas. The thermal noise is independent of the nature of the detected gas; it is a white noise. In Fig. 2, we have shown the shape of the plot of the frequency dependency of the PSD on the gas sensor noise.

\section{Gases Identification Method: Theoretical Approach}

The gas identification method that we propose is based on the product $f \cdot S(f)$, where $f$ is the frequency and $S(f)$ is the PSD of the gas sensor noise. In this section, this product will be mathematically studied. The aim of this study is to see if there is a theoretical correlation between the expression of this product and the nature of the detected gas.

Indeed, from Eqs. (3) and (4), the PSD of the total gas sensor noise, is the addition of two terms. The first term is the A-D noise which is a combination of Lorentzians. The second term is a white noise which corresponds to the thermal noise. Hence, the product that we propose to study is expressed by

- If the gas sensor resistance is biased by a voltage $V_{0}$ and the measured noise is a current fluctuation:

$$
f S_{\delta I_{\text {sensor }}}(f)=\frac{V_{0}^{2}}{R_{\text {sensor }}^{4}} \sum_{i=1}^{g} S_{i} \frac{f}{1+\left(\frac{f}{f_{c i}}\right)^{2}}+\frac{4 k T}{R_{\text {sensor }}} f .
$$

- If the gas sensor resistance is biased by a current $I_{0}$ and the measured noise is a voltage fluctuation:

$$
f S_{\delta V_{\text {sensor }}}(f)=I_{0}^{2} \sum_{i=1}^{g} S_{i} \frac{f}{1+\left(\frac{f}{f_{c i}}\right)^{2}}+4 k T R_{\text {sensor }} f .
$$

It is worth noting that, in both cases, the term which may have a correlation with the nature of the detected gas is the first term which contains the A-D noise (the combination of Lorentzians). Thus, in order to do a mathematical study of the product $f \cdot S(f)$, we can limit our study to the function expressed by:

$$
C(f)=\sum_{i=1}^{g} S_{i} \frac{f}{1+\left(\frac{f}{f_{c i}}\right)^{2}}=\sum_{i=1}^{g} C_{i}(f),
$$


where

$$
C_{i}(f)=S_{i} \frac{f}{1+\left(\frac{f}{f_{c i}}\right)^{2}} .
$$

In order to study the function $C(f)$, it is more convenient to begin our study by the elemental function $C_{i}(f)$ and look for a gas identification parameter. Then, once we find an identification parameter in case of one single elemental function $C_{i}(f)$, we can extend our analysis in case of a combination of $C_{i}(f)$.

By making a mathematical study on the variation of the function $C_{i}(f)$ when $f$ is a positive frequency, we can easily obtain the plot of the function $C_{i}(f)$ (see Fig. 3) and show that it reaches its maximum at the frequency $f_{c i}$ and this maximum is given by

$$
C_{i \max }=\frac{1}{2} S_{i} f_{c i}
$$

Since $S_{i}$ and $f_{c i}$ depend on the nature of the detected gas, we conclude that the maximum of each elemental function $C_{i}(f)$ depends on the nature of the detected gas.

Now, let's extend our mathematical analysis in the case of a combination of $C_{i}(f)$.

Equation (8) shows that the function $C(f)$ is a combination of elemental functions $\left(C_{i}(f)\right)_{1<i<g}$. Besides, we have just shown that each function $C_{i}(f)$ has a maximum $C_{i \max }$ at a frequency $f_{c i}$. Thus, using a mathematical analysis of Eq. (7), we can show (see Appendix) that the function $\mathrm{C}(f)$ has also a maximum $C_{\max }$ at a frequency $f_{0}$ which satisfies the following:

$$
\left\{\begin{array}{l}
f_{c 1}<f_{0}<f_{\text {cg }} \\
C_{\max }=C\left(f_{0}\right)
\end{array} .\right.
$$

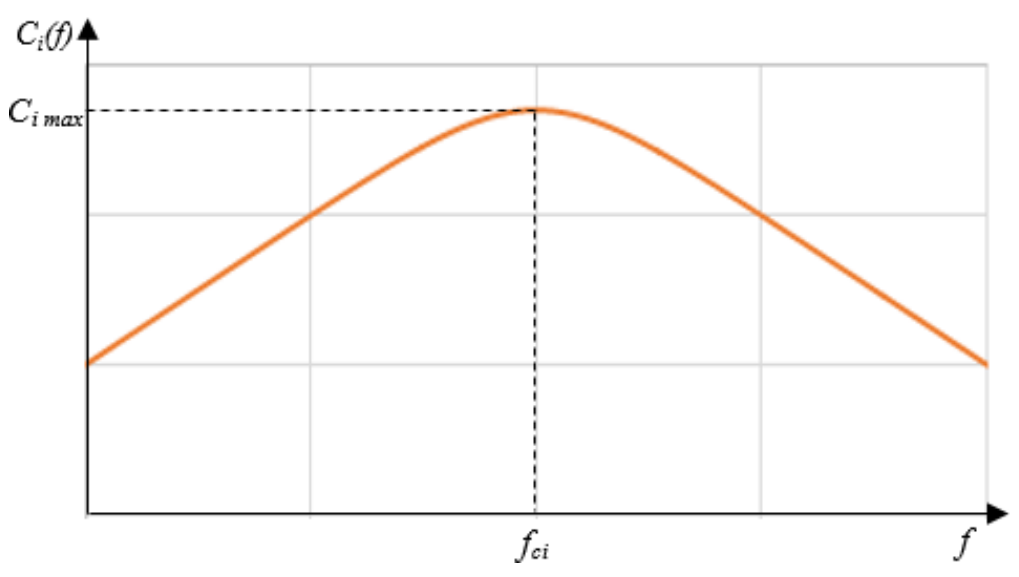

Fig. 3. Plot of the function $C_{i}(f)$ in logarithmic scale. 
Moreover, from Eq. (7), the function $C(f)$ can be mathematically considered as a parametric function which has $2 g$ parameters: the various noise levels $\left(S_{i}\right)_{1<i<g}$ and the cut-off frequencies $\left(f_{c i}\right)_{1<i<g}$, which are involved in the expression of the PSD of the gas sensor noise (see Eqs. (2) and (3)). Bearing in mind that all these parameters depend on the nature of the detected gas (see [18]), it is clear that the output of each input $f$ by this function depends on the nature of the detected gas. Thus, the maximum $\mathrm{C}_{\max }$ of the function $C(f)$, which is the output of $f_{0}$ by the function $C(f)$, depends on the nature of the detected gas. It is expressed by

$$
C_{\max }=C\left(f_{0}\right)=\sum_{i=1}^{g} S_{i} \frac{f_{0}}{1+\frac{f_{0}^{2}}{f_{c i}^{2}}} .
$$

Finally, we have shown that the function $C(f)$ has a maximum which depends on the nature of the detected gas. This theoretical result will be compared with experimental results in the following section.

In Figs. 4 and 5, we show a simulation of the function $C(f)$ taking as an example a combination of three functions $\left(L_{i}^{\prime}(f)\right)_{1<i<4}$

$$
C(f)=C_{1}(f)+C_{2}(f)+C_{3}(f) .
$$

The shape of the function $C(f)$ will depend on the values of the different maximums $\left(C_{1 \max }, C_{2 \max }\right.$ and $\left.C_{3 \max }\right)$ reached by the functions $C_{1}(f), C_{2}(f)$ and $C_{3}(f)$. We can resume all cases in two general cases:

- If all quantities $\left(S_{i} \cdot f_{c i}\right)_{1<i<3}$ are equal, then all $\left(C_{i \max }\right)_{1<i<3}$ are equal also. Thus, the function $C(f)$ will have a shape as shown in Fig. 4. That is the plot of the function has a maximum for one or more frequencies which are close to the cut-off frequencies $\left(f_{c i}\right)_{1<i<3}$

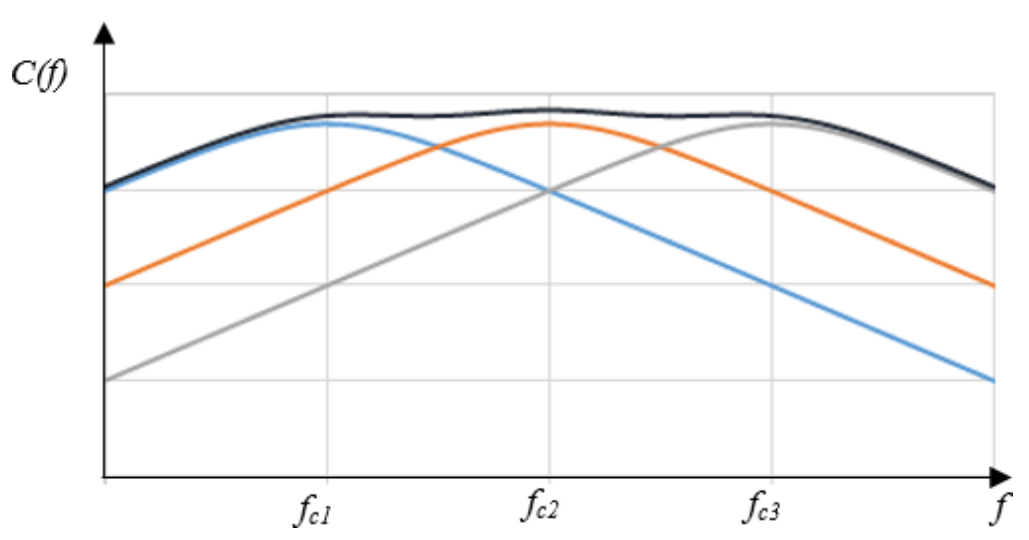

Fig. 4. Plot of the function $C(f)$ with $C_{1 \max }=C_{2 \max }=C_{3 \max }$. 


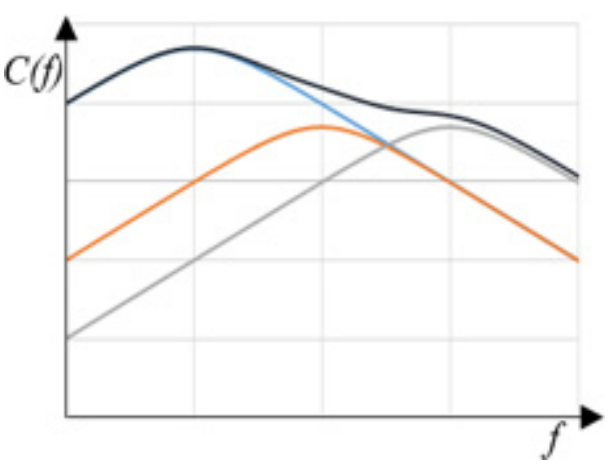

(a)

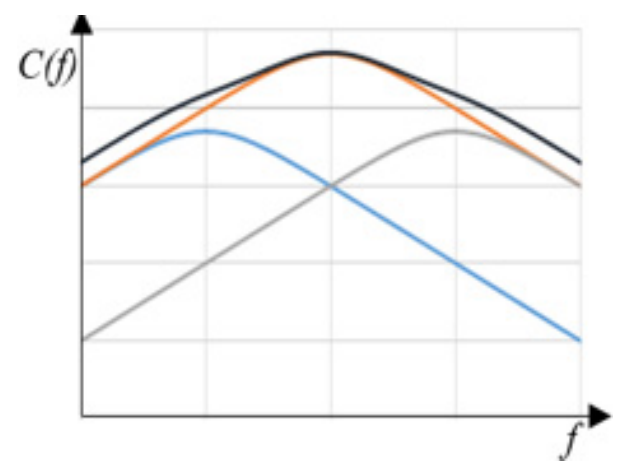

(b)

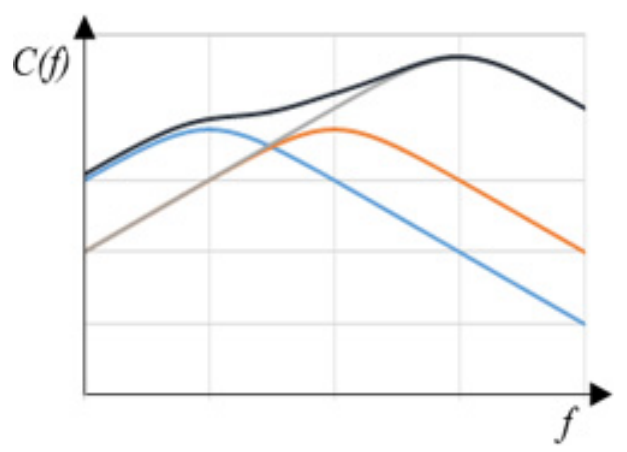

(c)

Fig. 5. Examples of possible plots of the function $C(f)$.

- If the quantities $\left(S_{i} \cdot f_{c i}\right)_{1<i<3}$ are not equal to each other (which means the maximums $\left(C_{i \max }\right)_{1<i<3}$ are not equal, too), then the function $C(f)$ will have a maximum at the frequency closest to that which gives the largest factor $S_{i} \cdot f_{c i}$. Three cases are given in Fig. 5:

- Case (a): $C_{1 \max }$ very large compared to $C_{2 \max }$ and $C_{3 \max }$, so $C(f)$ reaches its maximum at a frequency close to $f_{c 1}$.

- Case (b): $C_{2 \max }$ very large compared to $C_{1 \max }$ and $C_{3 \max }$, so $C(f)$ reaches its maximum at a frequency close to $f_{c 2}$.

- Case (c): $C_{3 \max }$ very large compared to $C_{2 \max }$ and $C_{1 \max }$, so $C(f)$ reaches its maximum at a frequency close to $f_{c 3}$.

It is worth noting that in Fig. 5, we do not represent all existing cases. We simply give three example of extreme cases. But, if for example we have two quantities $\left(S_{i} \cdot f_{c i}\right)$ and $\left(S_{k} \cdot f_{c k}\right)$ which have two values close but very large with respect to the third quantity, then the function $C(f)$ will have its maximum at the frequency closest to the average of the two frequencies $f_{c i}$ and $f_{c k}$. 


\section{Experimental Results}

\subsection{Experimental set up}

Noise measurements have been performed on metal-oxide gas micro sensors with $\mathrm{WO}_{3}$ sensing layer. $\mathrm{WO}_{3}$ thin films were prepared by reactive radio frequency (13.56 Mhz) magnetron sputtering, using a $99.9 \%$ pure tungsten target. The films (thickness $40 \mathrm{~nm}$ ) were sputtered on $\mathrm{SiO}_{2} / \mathrm{Si}$ substrates with platinum interdigitated electrodes, in a reactive atmosphere of oxygen-argon mixture. Figure 6(a) shows the typical geometric structure of the microsensor. The photo in Fig. 6(b) shows the top view of the actual microsensor. The microsensor (without integrated heating device) was placed on a heated plate, and biased by a voltage of $1 \mathrm{~V}$ applied between electrodes $E_{1}$ and $E_{2}$ shown in Fig. 6(b).

The experimental setup which we used to measure the sensor noise response in various gas is shown in Fig. 7.

The sensors are placed in a stainless steel measurement chamber where mixed gases can be admitted and evacuated via mass flow controllers and distribution valves. The gas chamber, the preamplifier, the sensors biasing and the power supply for sensor local heating are incorporated in a Faraday cage. As the resistance of the sensing layer is very high ( $\mathrm{M} \Omega$ range), and for best measurement conditions, the sensor is biased by a voltage source, and the fluctuation of the current crossing the sensing layer is amplified by a low noise current amplifier Stanford Research SR570 (LNA on Fig. 1). The PSD of the outputted voltage is recorded using a SR785 FFT Signal Analyzer, in the $0.1 \mathrm{~Hz}-100 \mathrm{kHz}$ range, at a sampling rate of 256,000 samples/s. The build in high pass filter of the SR570 amplifier was set to a cutoff frequency of $0.03 \mathrm{~Hz}$ with a $-12 \mathrm{~dB} /$ octave slope. Averaging was made on two successive measurements and the reproducibility of the spectra was checked. The noise spectrum of the measurement system was subtracted from the measured spectra to obtain the real spectra of the gas sensor. The measurement time is about $10 \mathrm{~min}$, mostly depending on the number of measurement points in the lowest frequency decades.

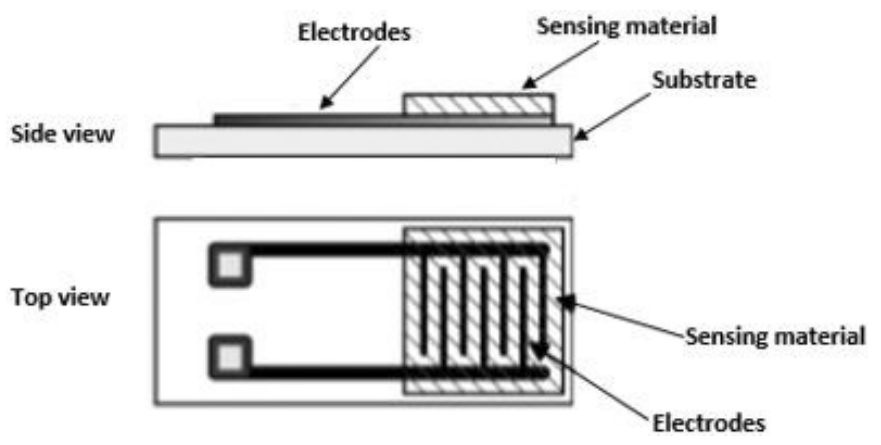

(a)

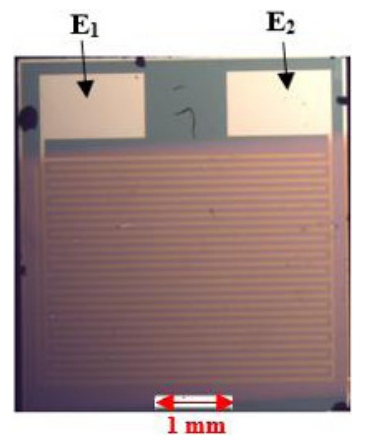

(b)

Fig. 6. A geometrical structure (a) and a real photo of the microsensor (b). 


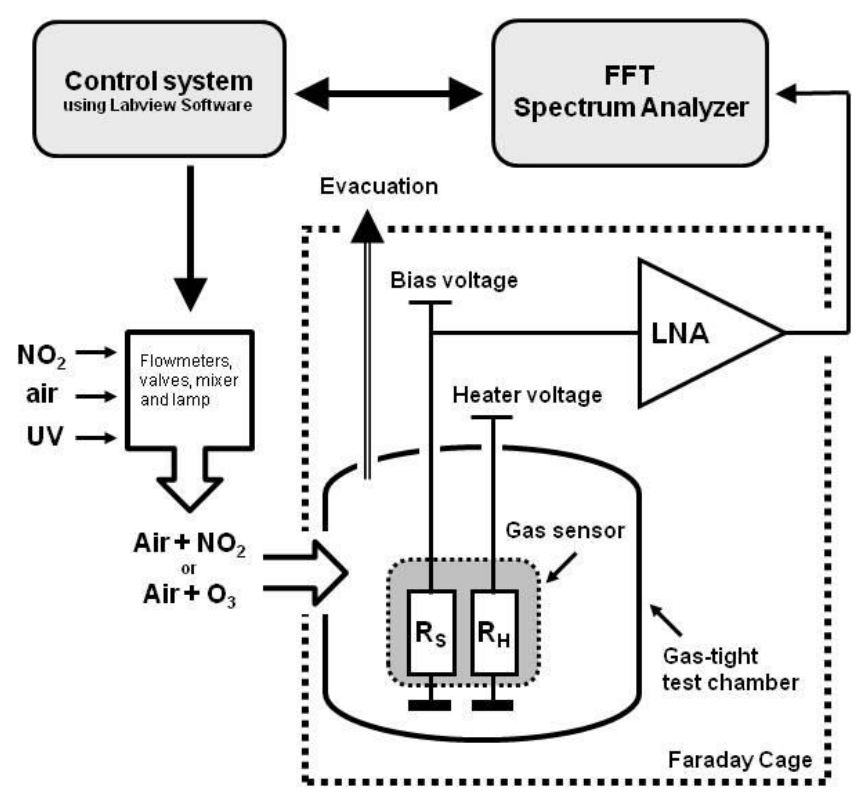

Fig. 7. General diagram of measurements setup used to characterize micro sensors noise responses.

For the present study, the PSD of the current fluctuations was recorded when the gas microsensor was exposed to nitrogen dioxide and ozone, diluted in dry air. An example is shown in Fig. 8.

Comparing the two noise spectra, one can see that they are quite different in the low frequencies range $(<100 \mathrm{~Hz})$ while nearly identical at frequencies greater than $100 \mathrm{~Hz}$. The white noise over $100 \mathrm{~Hz}$ is mainly the contribution of thermal noise due to the sensing layer resistance.

\subsection{Noise spectra parameters extraction}

According to "Eq. (3)", we have decomposed the low frequency part of the measured spectra into a sum of Lorentzians. In Fig. 9, it is clearly seen that each spectrum is well fitted by the sum of three Lorentzians and a white noise for the high frequency part.

That means the PSD of the A-D noise generated by the adsorption of either ozone or nitrogen dioxide, $S_{\delta I_{\mathrm{A}-\mathrm{D}}}$, is described by decomposition into three Lorentzians.

$$
S_{\delta I_{\mathrm{A}-\mathrm{D}}}(f)=\sum_{i=1}^{3} H_{i} \frac{1}{1+\left(\frac{f}{f_{c i}}\right)^{2}} .
$$

Using a modeling of all Lorentzians (see Fig. 8), we get an extraction of their parameters $\left(H_{i}\right.$ and $\left.f_{c i}\right)$ given in Table 1 .

As explained in Sec. 3, the PSD of the A-D noise generated by the gas adsorption $S_{\delta I_{\mathrm{A}-\mathrm{D}}}$ is proportional to the PSD of sensor resistance fluctuation $S_{\delta R_{\text {sensor }}}$. These 


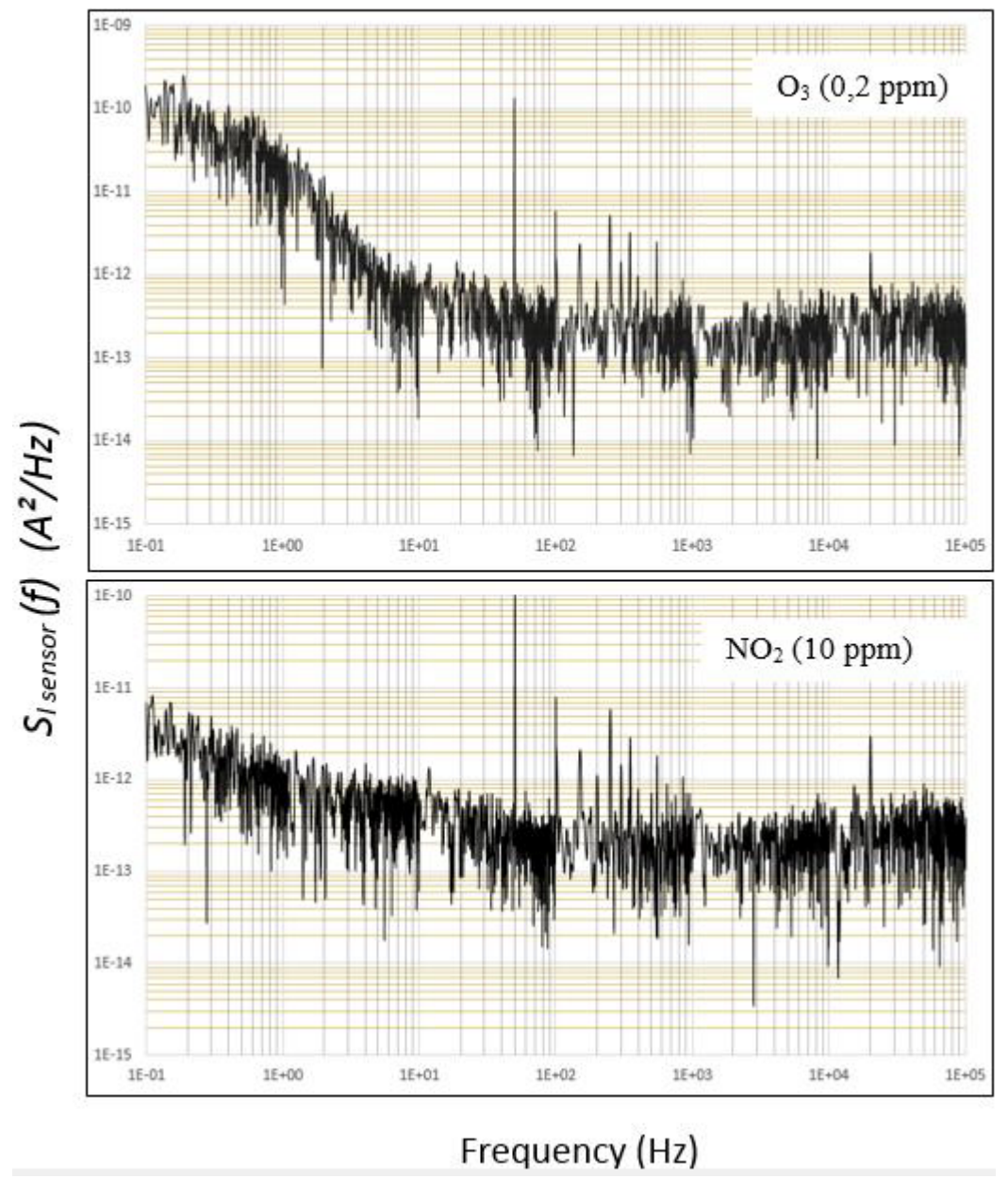

Fig. 8. Noise response under ozone $(0,2 \mathrm{ppm})$ and nitrogen dioxide $(10 \mathrm{ppm})$ in dry air.

power spectrum densities are linked by the following expression:

$$
S_{\delta I_{\mathrm{A}-\mathrm{D}}}(f)=\frac{V_{0}^{2}}{R_{\mathrm{sensor}}^{4}} S_{\delta R_{\text {sensor }}}(f) .
$$

Hence, using the decomposition of the current noise $S_{\delta I_{\mathrm{A}-\mathrm{D}}}$ given by curves in Fig. 8 and the relationship between $S_{\delta I_{\mathrm{A}-\mathrm{D}}}$ and $S_{\delta R_{\text {sensor }}}$ given by "Eq. (14)", we can confirm that the PSD of the sensing layer fluctuation generated by the adsorption of ozone and $\mathrm{NO}_{2}$ is described by a decomposition into three Lorentzians. Hence, the PSD of measured sensor resistance fluctuation is expressed by

$$
S_{\delta R_{\text {Sensor }}}(f)=\sum_{i=1}^{3} S_{i} \frac{1}{1+\left(\frac{f}{f_{c i}}\right)^{2}} .
$$




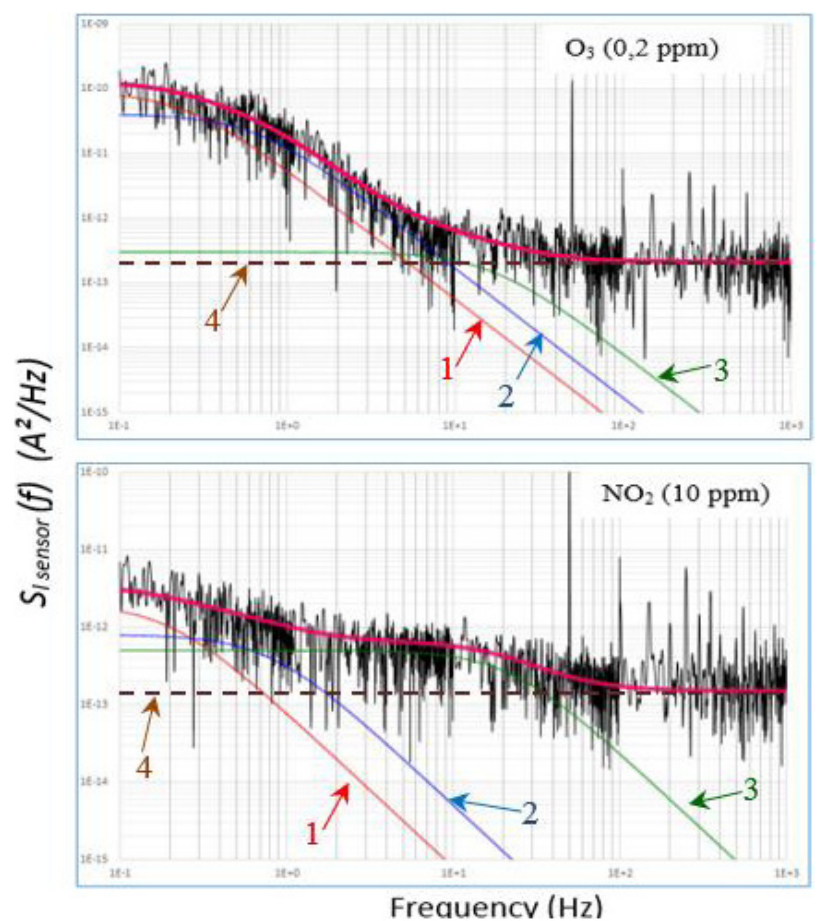

Fig. 9. Decomposition of noise response of the sensor under $0.2 \mathrm{ppm}$ of ozone and $10 \mathrm{ppm}$ of nitrogen dioxide in dry air. 1-3: Lorentzians constituting the low frequency part of the spectrum; dotted line 4: thermal noise; thick (red) line: fitted spectrum using "(Eq. (3))".

Table 1. Extracted noise parameters.

\begin{tabular}{lcc}
\hline Gas & $\mathrm{O}_{3}$ & $\mathrm{NO}_{2}$ \\
\hline $\mathrm{H}_{1}\left(\mathrm{~A}^{2} / \mathrm{Hz}\right)$ & $9 \cdot 10^{-11}$ & $2 \cdot 10^{-12}$ \\
$f_{c 1}(\mathrm{HZ})$ & 0,25 & 0,2 \\
$\mathrm{H}_{2}\left(\mathrm{~A}^{2} / \mathrm{Hz}\right)$ & $4 \cdot 10^{-11}$ & $8 \cdot 10^{-13}$ \\
$f_{c 2}(\mathrm{HZ})$ & 0,65 & 0,8 \\
$\mathrm{H}_{3}\left(\mathrm{~A}^{2} / \mathrm{Hz}\right)$ & $3 \cdot 10^{-13}$ & $5 \cdot 10^{-13}$ \\
$f_{c 3}(\mathrm{HZ})$ & 16,5 & 22 \\
Thermal noise $\left(\mathrm{A}^{2} / \mathrm{Hz}\right)$ & $2,1 \cdot 10^{-13}$ & $1,5 \cdot 10^{-13}$
\end{tabular}

Using "Eqs. (13) and (14)" we can see clearly that $S_{i}$ is proportional to the extracted value of $H_{i}$. It can be expressed by

$$
H_{i}=\frac{V_{0}^{2}}{R_{\mathrm{sensor}}^{4}} S_{i} .
$$

The values of cutoff frequencies shown in Table 1 are not clearly correlated to the nature of the detected gas. These experimental results seem to be inconsistent with 
our model which shows that the cut-off frequency depends on the nature of the detected gas by means of the molecule mass and size, and its adsorption energy [18]. However, the similarity between cut-off frequencies can be explained by the similarity between $\mathrm{O}_{3}$ and $\mathrm{NO}_{2}$ molecules. Indeed, the two molecules differ only by one atom $(\mathrm{O}$ replaced by $\mathrm{N}$ ), and these atoms are very close in mass (atomic weight: 16 and 14, respectively) and radius (radius of Van der Waals: $1.55 \AA$ and $1.6 \AA$, respectively [31]). To confirm the link between the cut-off frequencies and the nature of the detected gas, we will make noise measurements under gases having larger differences in terms of molecule sizes and mass, such as benzene. However, it is necessary to find a method to discriminate gases whose molecules are not very different. So, we will apply the method proposed above to the case of $\mathrm{O}_{3}$ and $\mathrm{NO}_{2}$.

\subsection{Experimental validation of the gases identification method}

In order to check the consistency of this gas identification method, we should apply it to our experimental results by plotting the frequency dependence of the measured quantity $f \cdot S_{I_{\text {sensor }}}(f)$ (the PSD of the measured gas sensor current fluctuation multiplied by the frequency).

Indeed, taking into account the contribution of thermal noise induced by the sensing layer resistance, the shape of the plot of the quantity $f \cdot S_{I_{\text {sensor }}}(f)$ will not have the same one given in simulation results given in Figs. 7 or 8 . In fact, the PSD of the gas sensor current noise is given by [18]

$$
S_{\delta I_{\text {sensor }}}(f)=S_{\delta I_{\mathrm{A}-\mathrm{D}}}(f)+\frac{4 k T}{R_{\text {sensor }}} .
$$

On the other hand, in Sec. 4.3, $S_{\delta I_{\mathrm{A}-\mathrm{D}}}(f)$ is expressed by a combination of three Lorentzians (see Table 1 ). Hence, the quantity $f \cdot S_{\delta I_{\text {sensor }}}(f)$ is expressed by the following:

$$
f S_{\delta I_{\text {sensor }}}(f)=\sum_{i=1}^{3} H_{i} \frac{f}{1+\left(\frac{f}{f_{c i}}\right)^{2}}+\frac{4 k T}{R_{\text {sensor }}} f .
$$

This expression shows that $f \cdot S_{\delta I_{\text {sensor }}}(f)$ is the sum of a first term which is a function of the type $C(f)$ studied above, and a second term which is a linear function of the frequency.

Thus, at low frequencies, the plot of the quantity $f \cdot S_{\delta I_{\text {sensor }}}(f)$ will show one of the shapes given by simulation results in Figs. 7 or 8 . At high frequencies, it will show a linear shape corresponding to the quantity $\frac{4 k T}{R_{\text {sensor }}} f$.

Figures 9 and 10 show the plot of $f \cdot S_{\delta I_{\text {sensor }}}(f)$, using experimental data for the values of $S_{\delta I_{\text {sensor }}}(f)$, in the case of ozone and nitrogen dioxide. Moreover, we have used the extracted values of Lorentzians' parameters from Table 1 to plot a simulation of the function $C(f)$.

Thus, at low frequency the plot of the quantity $f \cdot S_{\delta I_{\text {sensor }}}(f)$ will have the same shape as the one given by simulation results in Figs. 7 or 8 . However, at high 


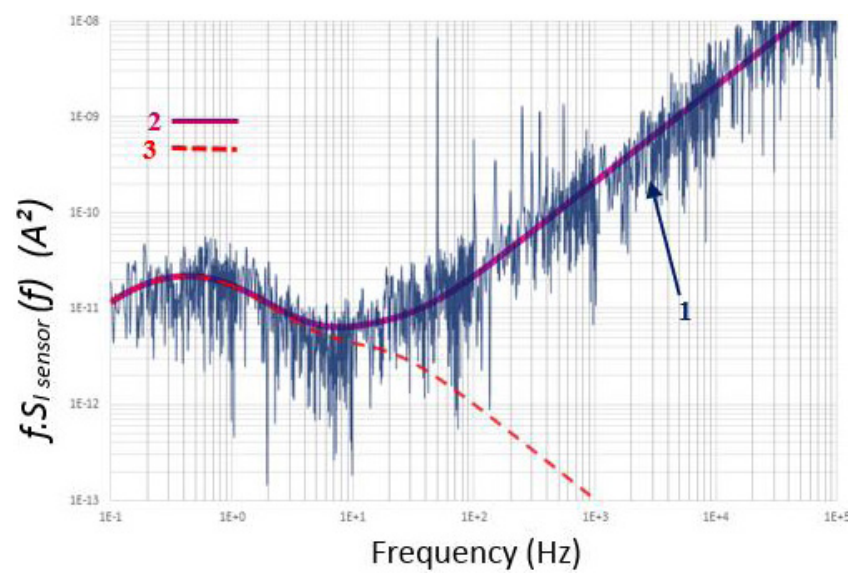

Fig. 10. Plot of the quantity $f \cdot S_{I_{\text {sensor }}}$ in the case of Ozone: 1 experimental; 2: modeling; 3: $C(f)$.

frequencies, it will have the shape of a linear curve which corresponds to the quantity $\frac{4 k T}{R_{\text {sensor }}} f$.

In Figs. 10 and 11, we plot the experimental quantity $f \cdot S_{I_{\text {sensor }}}(f)$ using experimental results for the values of $\left.S_{I_{\text {sensor }}}(f)\right)$ in case of two different gases: ozone and nitrogen dioxide. Moreover, we have used the extracted values of Lorentzians parameters from Table 1 and plotted a simulation of the quantity $f \cdot S_{I_{\text {sensor }}}(f)$. Besides, in order to compare the theoretical result obtained in Sec. 3, we have plotted the quantity $f\left(S_{\text {Isensor }}-\frac{4 k T}{R_{\text {sensor }}}\right)$ which corresponds to the function $C(f)$.

Figures 10 and 11 show, at high frequencies, the presence of a linear curve as predicted by "Eq. (18)".

However, at low frequencies, we note many differences between plots of $f \cdot S_{\delta I_{\text {sensor }}}(f)$. Indeed, in the case of ozone, at low frequencies, the plot of the measured

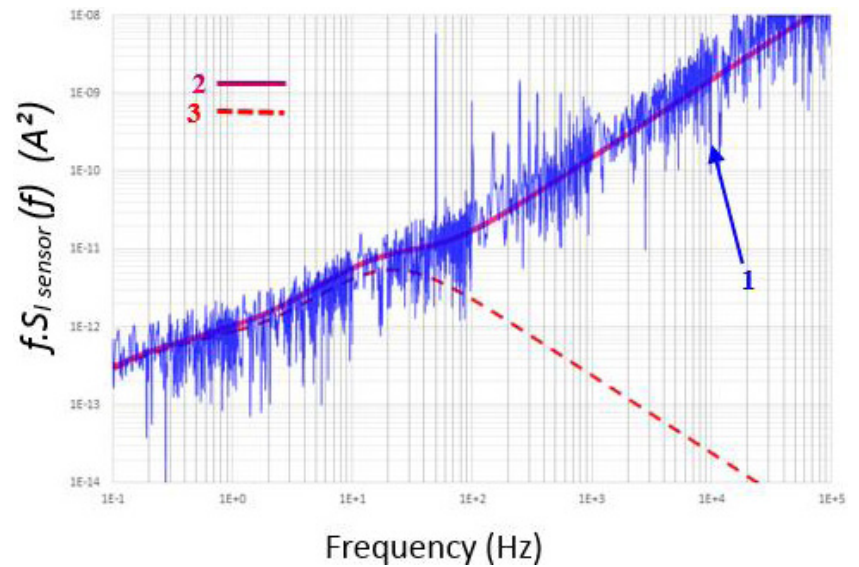

Fig. 11. Plot of the quantity $f \cdot S_{I_{\mathrm{sensor}}}$ in the case of $\mathrm{NO}_{2}: 1$ experimental; 2:modeling; $3: C(f)$. 
quantity $f \cdot S_{\delta I_{\text {sensor }}}(f)$ has the shape given by simulation in Fig. 5 case (a). In the case of nitrogen dioxide, we see that, at low frequencies, the plot of the measured quantity $f \cdot S_{I_{\text {sensor }}}(f)$ has the shape given by simulation in Fig. 5, Case (c). In fact, this is explained as follows:

- In the case of nitrogen dioxide, we have

$S_{1} \cdot f_{c 1}=4 \cdot 10^{-13}, S_{2} \cdot f_{c 2}=6,4 \cdot 10^{-13}$ and $S_{3} \cdot f_{c 3}=110 \cdot 10^{-13}$ which corresponds to the case c) in Fig. 5 that means the quantity $f \cdot S_{\delta I_{\mathrm{AD}}}(f)$ must have its maximum at the frequency close to the frequency $f_{c 3}$.

- In the case of ozone, we have:

$S_{1} \cdot f_{c 1}=2,5 \cdot 10^{-11}, \quad S_{2} \cdot f_{c 2}=2,6 \cdot 10^{-11}$ and $S_{3} \cdot f_{c 3}=49,5 \cdot 10^{-13}$ that is $S_{1} \cdot f_{c 1 \approx} S_{2} \cdot f_{c 2}$ and $S_{1} \cdot f_{c 1}>S_{3} \cdot f_{c 3}$, which corresponds to the case explained in the paragraph just below Fig. 8, that means the quantity $f \cdot S_{\delta I_{\mathrm{AD}}}(f)$ must have its maximum at the frequency closest the average of the two frequencies $f_{c 1}$ and $f_{c 2}$.

To confirm the latter analysis, we have plotted in Figs. 9 and 10 (curve 3) the quantity $f\left(S_{I_{\text {sensor }}}(f)-S_{\text {th }}\right)$, where $S_{\text {th }}$ is the thermal noise converted to current noise. We have subtracted the thermal noise in order to better see the frequency dependency of $f \cdot S_{\delta I_{\mathrm{A}-\mathrm{D}}}(f)$. Hence, the latter analysis is confirmed by the curves 3 in Figs. 9 and 10.

Thus, we have shown that the quantity $f \cdot\left(S_{I_{\text {sensor }}}(f)-S_{\text {th }}\right)$ has a maximum which is characteristic of the nature of the detected gas. Detecting this maximum is a sensitive method to identify a gas.

\section{Discussion}

From noise measurements on $\mathrm{WO}_{3}$ gas sensors, we showed that the PSD of the gas sensor noise is a combination of Lorentzians having a low frequency magnitude and a cut-off frequency which depends on the nature of the detected gas. The dependence of model coefficients with the nature of gas is consistent with the theory of A-Dnoise we have developed [18]. Furthermore, an experimental work on $\mathrm{WO}_{3}$ semiconductor in air [28] showed the existence of a Lorentzian contribution to the measured noise spectra.

However, noise measurements on $\mathrm{SnO}_{2}$ sensors [27] revealed a $1 / f^{\alpha}$ frequency dependence. As discussed by the authors of [27], this flicker noise is a function of sensor resistance and of other unknown parameters. Moreover, resistance fluctuations measured on two types of gas sensors TGS 826 and TGS 825 [29] under ammonia and hydrogen sulfide have given a $1 / f$ noise spectra. It is worth noting that the multi-Lorentzian noise spectrum given by our previous model of $\mathrm{A}-\mathrm{D}$ noise (see Eq. (1)) is not inconsistent with these experimental results since we have shown in [18] that, according to the sensing layer microstructure (distribution of grain sizes in the sensing layer), a $1 / f^{\alpha}$ spectrum can result from the superposition of a finite 
number of Lorentzian spectra in MOX gas sensor. Moreover, similar result was shown in the case of thin film resistors [30].

In the second part of this paper, we presented a new noise spectroscopy-based method for discriminating gases. It is based on the product $f \cdot S(f)$, where $f$ is the frequency and $S(f)$ is the power density spectrum of the gas sensor noise. In fact, as it was mentioned in the introduction, this method has been used in many works [3]-[12]. But what makes the differences between all these works is the parameter that is used for gas recognition. For example, in [11], the average slope of the $f \cdot S(f)$ product, fixed for different decades of frequency $f$, has been proposed as one of the possible constitutive parameters for gas recognition. In [12], the parameter used as a gas signature is the characteristic frequency at which the product $f \cdot S(f)$ reaches its maximum. In this paper we have presented a new parameter which can be used as a gas signature based on the product $f \cdot S(f)$. This parameter is the maximum of the product $f \cdot S(f)$. Furthermore, what makes the originality of this study is that the dependency of this new parameter as a function of the nature of detected gas is theoretically shown and experimentally proved.

By plotting the frequency dependency of the measured quantity $f \cdot S(f)$, in the case of nitrogen dioxide and ozone, we showed that the product $f \cdot S(f)$ has a specific maximum for each gas. However, the application of the same method on $\mathrm{SnO}_{2}$ sensor [11], in the case of clean air, methanol and acetone, revealed that the plot of the product $f \cdot S(f)$ has a wide plateau over two frequency decades followed by a decrease. This difference with our experimental results can be explained by the differences in the sensing films. If the plot of the product $f \cdot S(f)$ presents a plateau on some frequency band, then one can easily deduce that the PDS of the sensor noise $S(f)$ has a $1 / f$ shape on that frequency band. Moreover, the possibility of obtaining an A-D noise with a $1 / f$ shape is linked to the distribution function of the grain sizes on the sensing layer surface [16]. Such a spectrum shape can be obtained if the A-D noise comes from the A-D process on a large number of different grain sizes uniformly distributed. Thus, this method can be considered as a useful tool that can give information on the underlying statistics of defects and grains forming the sensing layer.

Considering our work and those of $[11,12,21]$, we can deduce that the gases discriminating method based on the product $f \cdot S(f)$ can be used in several ways. If the plot of this product presents a plateau shape, then it is more convenient to use the slope of the decreasing part of the plot as a gas signature, as it was applied in [11, 21]. In other cases, when the plot shows a peak, another parameter can be used such as the characteristic frequency at which the peak occurs [12] or the maximum of the product $f \cdot S(f)$, as it was presented in this work.

\section{Conclusion}

In this work, we propose a new method for obtaining a FES fingerprint of a gas using a single gas micro sensor. Starting from our model of A-D noise in MOX sensors 
previously developed, we show theoretically that the product of frequency by the PSD of the gas sensing layer resistance fluctuations often has a maximum which is characteristic of the gas. This property was experimentally confirmed in the case of the detection of $\mathrm{NO}_{2}$ and $\mathrm{O}_{3}$ using a $\mathrm{WO}_{3}$ sensing layer. This method could be useful for classifying gases.

Furthermore, our noise measurements confirm our previous model showing that PDS of the A-D noise in MOX gas sensor is a combination of Lorentzians having a low frequency magnitude and a cut-off frequency which depends on the nature of the detected gas.

In future work, we plan to use this method for obtaining the FES signature of other gases such as $\mathrm{CO}, \mathrm{NH}_{3}$, ethanol, benzene..., and analyzing mixtures of these gases, in order to determine also the limit of this method. Another future direction would be to explore experimentally the link between the number of Lorentzians composing the low frequencies part of the PSD of MOX micro sensors and the distribution of grain sizes in the sensing layer.

\section{Acknowledgments}

This research did not receive any specific grant from funding agencies in the public, commercial, or not-for-profit sectors.

\section{Appendix A}

- How to show that the function $C(f)$ has a maximum $C_{\max }$ and that this maximum is reached at a frequency $f_{0}$ which satisfies

$$
f_{c 1}<f_{0}<f_{c g} .
$$

In order to show that the function $C(f)$ has a maximum, we have to make a mathematical study of this function.

The expression of the function $C(f)$ is given by

$$
C(f)=\sum_{i=1}^{g} f \cdot L_{i}(f)=\sum_{i=1}^{g} C_{i}(f),
$$

where

$$
C_{i}(f)=S_{i} \frac{f}{1+\left(\frac{f}{f_{c i}}\right)^{2}} .
$$

In order to study $C(f)$, we have to study the sign of its first derivative.

The first derivative of $C(f)$ is expressed by the following:

$$
C^{\prime}(f)=\frac{d C}{d f}(f)=\sum_{i=1}^{g} \frac{d C_{i}}{d f}(f)=\sum_{i=1}^{g} C_{i}^{\prime}(f),
$$


where

$$
C_{i}^{\prime}(f)=S_{i} \frac{1-\frac{f^{2}}{f_{c i}^{2}}}{\left[1+\frac{f^{2}}{f_{c i}^{2}}\right]^{2}} .
$$

From Eq. (A.4), we can easily deduce that:

$$
\left\{\begin{array}{lll}
\text { for each } \quad f<f_{c i} ; & C_{i}^{\prime}(f)>0 \\
& & C_{i}^{\prime}\left(f_{c i}\right)=0 \\
\text { for each } \quad f>f_{c i} ; & C_{i}^{\prime}(f)<0
\end{array}\right.
$$

Let $\left(f_{c 1}, f_{c 2}, f_{c 3}, \ldots, f_{c g}\right)$ be the various cut off frequencies involved in the expression of the PSD of the gas sensor noise (see "Eq. (2)" in the paper), arranged in the following way:

$$
f_{c 1}<f_{c 2}<f_{c 3}<\cdots<f_{c g}
$$

- Let's study the sign of $C^{\prime}(f)$ for all frequencies $f$ satisfying: $0 \leq f<f_{c 1}$ From (A.5) we deduce that for each frequency $f<f_{c 1}$, we have

$$
\left\{\begin{array}{l}
C_{1}^{\prime}(f)>0 \text { since } f<f_{c 1} \\
C_{2}^{\prime}(f)>0 \quad \text { since } f<f_{c 1}<f_{c 2} \\
C_{3}^{\prime}(f)>0 \quad \text { since } f<f_{c 1}<f_{c 2}<f_{c 3} \\
\cdots \cdots \\
\cdots \cdots \\
C_{g}^{\prime}(f)>0 \quad \text { since } f<f_{c 1}<f_{c 2}<f_{c 3}<\cdots<f_{c g}
\end{array}\right.
$$

By adding all the $g$ inequalities in "Eq. (A.7)" we deduce that:

$$
C_{1}^{\prime}(f)+C_{2}^{\prime}(f)+C_{3}^{\prime}(f)+\cdots+C_{g}^{\prime}(f)>0
$$

which means $C^{\prime}(f)>0$.

Hence, we deduce that:

$$
\text { for each } f<f_{c 1} \text {; we have: } C^{\prime}(f)>0
$$

- Let's study the sign of $C^{\prime}(f)$ for each frequency $f$ satisfying: $f_{c 1}<f$ ?

From (A.5) we can confirm that for each frequency $f>f_{c g}$ we have:

$$
\begin{cases}C_{g}^{\prime}(f)<0 & \text { since } f>f_{c g} \\ C_{g-1}^{\prime}(f)<0 & \text { since } f>f_{c g}>f_{c g-1} \\ \cdots \cdots & \\ \cdots \cdots & \text { since } f>f_{c g}>f_{c g-1}>\cdots>f_{c 3} \\ C_{3}^{\prime}(f)<0 & \text { since } f>f_{c g}>f_{c g-1}>\cdots>f_{c 3}>f_{c 2} \\ C_{2}^{\prime}(f)<0 & \text { since } f>f_{c g}>f_{c g-1}>\cdots>f_{c 3}>f_{c 2}>f_{c 1} \\ C_{1}^{\prime}(f)<0 & \end{cases}
$$


By adding all the $g$ inequalities in (A.9) we deduce that

$$
\text { for each } f>f_{c g} ; \quad C^{\prime}(f)<0 .
$$

Finally, from (A.8) and (A.10) we conclude that:

$$
\left\{\begin{array}{lll}
\text { for each } & f<f_{c 1} ; & C^{\prime}(f)>0 \\
\text { for each } & f>f_{c g} ; & C^{\prime}(f)<0
\end{array}\right. \text {. }
$$

Hence, we can conclude that:

- For each frequency $f$ satisfying $0 \leq f<f_{c 1}$, the function $C(f)$ is increasing.

- For each frequency $f$ satisfying $f_{c g}<f$, the function $C(f)$ is decreasing.

Besides, using (A.2) we deduce that:

$$
\left\{\begin{array}{c}
C(0)=\sum_{i=1}^{g} C_{i}(0)=0 \\
\lim _{f \rightarrow+\infty}[C(f)]=0
\end{array} .\right.
$$

Hence, using (A.11) and (A.12), we obtain the first part of the curve of the function $C(f)$ (Fig. A.1)

Since the curve increases in the vicinity of $f_{c 1}$ and decreases in the vicinity of $f_{c g}$, so after passing through the abscissa point $\mathrm{f}_{c 1}$ the curve must pass through a maximum to return to the abscissa point $f_{c g}$. Hence, we conclude that it exists at a frequency $f_{0}$ which satisfies

$$
\left\{\begin{array}{l}
f_{c 1}<f_{0}<f_{c g} \\
C(f) \leq C\left(f_{0}\right) \quad \text { for each } f \geq 0
\end{array} .\right.
$$

The value of $C\left(f_{0}\right)$ is the maximum of $C(f)$, it will be noted as $C_{\max }$.

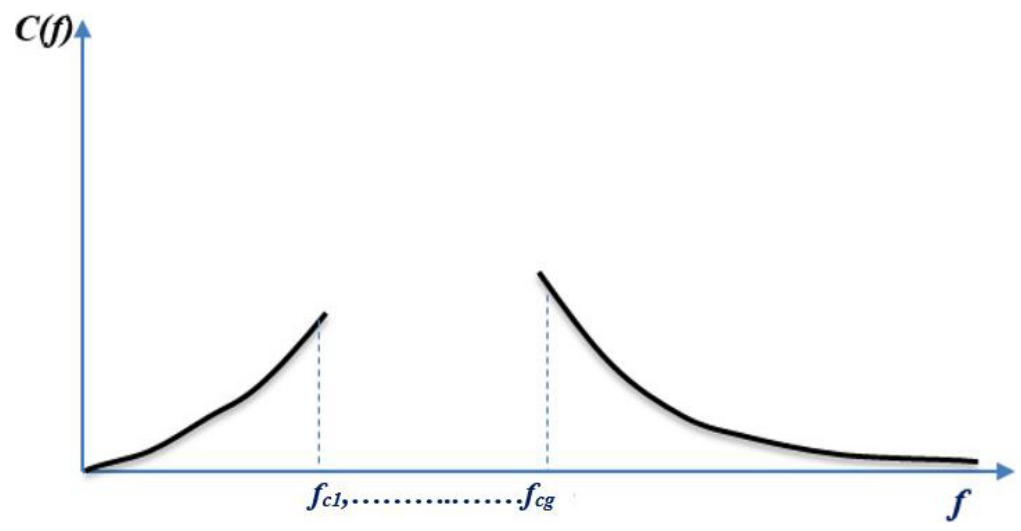

Fig. A.1. First part of the plot of $C(f)$. 


\section{References}

[1] N. Barsan and U. Weimar, Conduction model of oxide gas sensors, J. Electroceram 7 (2001) 143-167.

[2] A. Ponzoni, C. Baratto, N. Cattabiani, M. Falascon, V. Galstyan, E. Nunez-Carmona, F. Rigoni, V. Sberveglieri, G. Zambotti and D. Zappa, Metal oxide gas sensors, a survey of selectivity, Sensors 17 (2017) 714.

[3] F. Gasparyan, H. Khondkaryan and M. Aleksanyan, New applications of the noise spectroscopy for hydrogen sensors, J. Mod. Phys. 5 (2014) 1662-1669.

[4] L. Lentka, J. M. Smulko, R. Ionescu, C. G. Granqvist and L. B. Kish, Determination of gas mixture components using fluctuation enhanced sensing and the LS-SVM regression algorithm, Metrol. Meas. Syst. 22(3) (2015) 341-350.

[5] L. B. Kish, R. Vajtai and C.-G. Granqvist, Extracting information from noise spectra of chemical sensors: Single sensor electronic noses and tongues, Sens. Actuators B 71 (2000) $55-59$.

[6] J. Smulko, C. G. Granqvist and L. B. Kish, On the statistical analysis of noise in chemical sensors and its application for sensing, Fluct. Noise Lett. 1 (2001) L147.

[7] A. K. Vidybida, Adsorption-desorption noise can be used for improving selectivity, Sens. Actuators A 107 (2003) 233-237.

[8] G. Schmera, C. Kwan, P. Ajayan, R. Vajtai and L. B. Kish, Fluctuation-enhanced sensing: Status and perspectives, IEEE Sens. J. 8(6) (2008) 714-719.

[9] C. Kwan, G. Schmera, J. Smulko, L. B. Kish, P. Heszler and C. Granqvist, Advanced agent identification with fluctuation-enhanced sensing, IEEE Sensors J. 8(6) (2008) 706-713.

[10] G. Schmera and L. B. Kish, Fluctuation-enhanced gas sensing by surface acoustic wave devices, Fluct. Noise Lett. 2 (2002) L117-L123.

[11] B. Ayhan, C. Kwan, J. Zhou, L. B. Kish, K. D. Benstein, P. H. Rogers and S. Semancik, Fluctuation enhanced sensing (FES) with a nanostructured, semiconducting metal oxide film for gas detection and classification, Sens. Actuators B 188 (2013) 651-660.

[12] S. Rumyantsev, G. Liu, R. A. Potyrailo, A. Balandin and M. S. Shur, Selective sensing of individual gases using graphene devices, IEEE Sensors J. 13(8) (2013) 2818-2822.

[13] J. X. Fang, W. H. Marlow, J. X. Lu and R. R. Lucchese, Monte Carlo studies of the effects of substrate size on the water-substrate interaction energy and water structure, J. Chem. Phys. 107 (1997) 5212-5216.

[14] Z. Djuric, O. Jaksic and D. Randjelovic, Adsorption-desorption noise in micromechanical resonant structures, Sens. Actuators A 96 (2002) 244-251.

[15] S. Gomri, J. L. Seguin and K. Aguir, Modelling on oxygen-chemisorption —induced noise in metallic oxide gas sensors, Sens. Actuators B 107 (2005) 722-729.

[16] S. Gomri, J. L. Seguin, J. Guerin and K. Aguir, Adsorption-desorption noise in gas sensors: Modelling using Langmuir and Wolkenstein models for adsorption, Sens. Actuators B 114 (2006) 451-459.

[17] S. Gomri, J. L. Seguin, J. Guerin and K. Aguir, A mobility and free carriers density fluctuations based model of adsorption-desorption noise in gas sensor, J. Phys. D, Appl. Phys. 41 (2008) 065501.

[18] S. Gomri, T. Contaret, J. Seguin, K. Aguir and M. Masmoudi, Noise modeling in MOX gas sensors, Fluct. Noise Lett. 16(2) (2017) 1750013.

[19] P. Bruschi, A. Nannini and B. Neri, Gas and vapor effects on the resistance fluctuation spectra of conducting plymer thin folm resistors, Sens. Actuators B 19 (1994) 421-425.

[20] G. Schmera and L. B. Kish, Surface diffusion enhanced chemical sensing by surface acoustic waves, Sens. Actuators B 93 (2003) 159-163. 
[21] J. L. Solis, L. B. Kish, R. Vajtai, C.-G. Granqvist, J. Olsson, J. Schnvrer and V. Lantto, Identifying natural and artificial odours through noise analysis with a sampling-and-hold electronics nose, Sens. Actuators B 77 (2001) 312-315.

[22] N. Yamazoe and K. Shimanoe, Overview of gas sensor technology, in Science and Technology of Chemiresistor Gas Sensors, eds. D. K. Aswal and S. K. Gupta (Nova Science Publishers, Inc, New York, 2007), pp. 1-31.

[23] T. Wolkenstein, Electronic Processes on Semiconductor Surfaces during Chemisorption (Consultants Bureau, New York, 1991).

[24] A. Rothschild and Y. Komen, Numerical computation of chemisorption isotherms for device modelling of semiconductor gas sensors, Sens Actuators B 93 (2003) 362-369.

[25] X. Wang, S. S. Yee and W. P. Carey, Transition between neck-controlled and grain boundary-controlled sensitivity of metal-oxide sensors, Sens. Actuators B 24-25 (1995) $454-457$.

[26] C. M. Van Vliet, Responsitivity and noise in illustrative solid-state chemical sensors, Sens. Actuators B 24-25 (1995) 6-16.

[27] J. M. Parks, B. T. Marquis, G. P. Harlmer and R. F. Schmitt, Resistance Noise Spectroscopy of $\mathrm{SnO}_{2}$ Thik Film Gas Sensor, Sensor Research and Development Corporation/ IEEE, Orono, Maine, USA (2000) 415-420.

[28] A. Hoel, L. K. J. Vandamme, L. B. Kish and E. Olsson, Current and voltage noise in $\mathrm{WO}_{3}$ nanoparticles films, J. Appl. Phys. 91(8) (2002) 15.

[29] M. Kotarski and J. Smulko, Noise measurement set-up for fluctuations-enhanced gas sensing, Metrol. Meas. Syst. XVI(3) (2009) 457-464.

[30] S. Hashingushi, Y. Yamagishi, T. Fukuda, M. Okhi, J. Sikula, P. Vasina, Generation of $1 / \mathrm{f}$ noise spectrum by relaxation process in thin film resistor, Qual. Reliab. Eng. Int. 14 (1998) 69-71.

[31] S. S. Batsanov, Van der Waals Radii of elements, Inorg. Mater. 37(8) (2001), 871-885. 